\title{
Publisher Correction: Carrier density and disorder tuned superconductor-metal transition in a two- dimensional electron system
}

\author{
Zhuoyu Chen (10) 1,2,3, Adrian G. Swartz ${ }^{1,2,3}$, Hyeok Yoon ${ }^{1,2}$, Hisashi Inoue ${ }^{1,2,3}$, Tyler A. Merz ${ }^{1,2}$, Di Lu³,4, \\ Yanwu Xie ${ }^{1,2,3}$, Hongtao Yuan ${ }^{1,3}$, Yasuyuki Hikita ${ }^{1,3}$, Srinivas Raghu ${ }^{1,3,4}$ \& Harold Y. Hwang ${ }^{1,2,3}$
}

Correction to: Nature Communications https://doi.org/10.1038/s41467-018-06444-2; published online 1 October 2018

The original HTML version of this Article omitted to list Harold Y. Hwang as a corresponding author and incorrectly listed Adrian G. Swartz as a corresponding author. This has been corrected in the HTML version of the Article. The PDF version was correct from the time of publication.

Published online: 29 October 2018

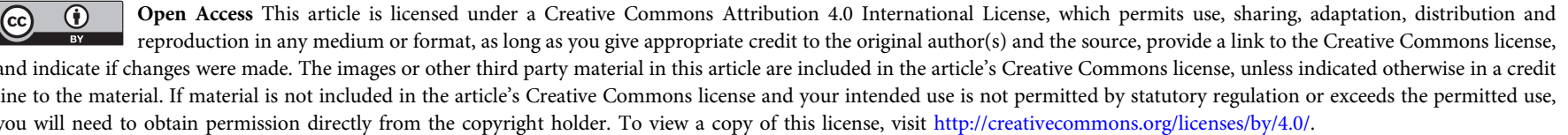
you will need to obtain permission directly from the copyright holder. To view a copy of this license, visit http://creativecommons.org/licenses/by/4.0/.

() The Author(s) 2018

\footnotetext{
${ }^{1}$ Geballe Laboratory for Advanced Materials, Stanford University, Stanford, CA 94305, USA. ${ }^{2}$ Department of Applied Physics, Stanford University, Stanford CA 94305, USA. ${ }^{3}$ Stanford Institute for Materials and Energy Sciences, SLAC National Accelerator Laboratory, Menlo Park, CA 94025, USA. ${ }^{2}$ Department of Physics, Stanford University, Stanford, CA 94305, USA. Correspondence and requests for materials should be addressed to

Z.C. (email: zychen@stanford.edu) or to H.Y.H. (email: hyhwang@stanford.edu)
} 